\title{
Gastrointestinal Malignancies and Venous Thromboembolic Disease: Clinical Significance and Endovascular Interventions
}

\author{
Xin Li, MD ${ }^{1}$ Sasan Partovi, MD ${ }^{1}$ Sameer Gadani, MD ${ }^{1} \quad$ Charles Martin III, MD $\quad$ Avi Beck, MD ${ }^{1}$ \\ Suresh Vedantham, MD²
}

${ }^{1}$ Section of Interventional Radiology, Imaging Institute, Cleveland Clinic Main Campus, Cleveland, Ohio

2 Section of Interventional Radiology, Mallinckrodt Institute of Radiology, Washington University in St. Louis, St. Louis, Missouri

Address for correspondence Sasan Partovi, MD, Section of Interventional Radiology, Imaging Institute, Cleveland Clinic Main Campus, 9500 Euclid Avenue, Cleveland, $\mathrm{OH} 44195$ (e-mail: partovs@ccf.org; sxp509@case.edu).

Dig Dis Interv 2020;4:260-266.

\begin{abstract}
Keywords

- CDT

- PCDT

- Gl cancer

- IVC filter

- anticoagulation

Gastrointestinal malignancy encompasses a wide range of disease processes. Its incidence and mortality rate rank among the highest of all cancers. Venous thromboembolic disease is a common complication of gastrointestinal malignancy. Anticoagulation remains the first-line therapy. However, for patients who cannot tolerate or have failed anticoagulation, inferior vena cava (IVC) filter placement may be an option. Furthermore, to improve symptom resolution and reduce the severity of postthrombotic syndrome, catheter-directed thrombolysis (CDT) may be an option. Recent randomized trials including the ATTRACT (Acute Venous Thrombosis: Thrombus Removal with Adjunctive Catheter-Directed Thrombolysis) trial have shed new light on the efficacy and safety of CDT and related methods. Overall, the decision to proceed with IVC filter placement or CDT must be individualized.
\end{abstract}

Gastrointestinal (GI) cancer is estimated to occur in more than 333,000 Americans and resulted in 167,790 cancerrelated death in $2020 .^{1}$ GI cancer encompasses a wide range of pathologies, including esophageal, gastric, pancreatic, neuroendocrine, hepatobiliary, colorectal, and anal cancers. $^{2-7}$ The estimated death associated with pancreatic, hepatobiliary, and colon cancers ranks among the top five of all malignancies. Additionally, GI cancer and its treatment are associated with multiple morbidities, including bleeding, infection, and thromboembolic diseases. Among those, venous thromboembolism (VTE) is a common complication that is six times more prevalent in the GI cancer population compared with the general population. ${ }^{8}$ In addition, VTE can result in recurrent deep venous thrombosis (DVT), pulmonary embolism (PE), and postthrombotic syndrome (PTS), which can lead to increased short-term mortality and longterm morbidity. Therefore, it is important to understand the received

June 19, 2020 accepted after revision July 27,2020 published online

September 22, 2020 prevalence and management of thromboembolic disease in GI cancer patients. In this article, we will discuss the clinical significance and management options of GI cancer-related thrombotic disease.

\section{Clinical Significance and Pathophysiology}

\section{Clinical Significance}

Cancer is associated with an increased risk of VTE. In comparison to the general public, the incidence of VTE in cancer patients is markedly higher. In a recent matched cancer versus noncancer patient cohort study, cancer patients had a hazard ratio of 4.7 for the occurrence of VTE and an incidence rate of 13.9 cases per 1,000 patient-years. ${ }^{9}$ With regard to GI cancer, the VTE incidences for esophageal, gastric, colon, and liver cancers were $12.5,15.4,13.4$, and 7.2 events per 1,000 patients, respectively. Pancreatic cancer had
Copyright $\odot 2020$ by Thieme Medical Publishers, Inc., 333 Seventh Avenue, New York, NY 10001, USA. Tel: +1(212) 760-0888.
DOI https://doi.org/ 10.1055/s-0040-1716739. ISSN 2472-8721.
Approach to Gastrointestinal
Malignancies; Guest Editors, Nicholas

Fidelman, MD, and R. Peter Lokken, MD 
a particularly high VTE incidence of 22.7 events per 1,000 patients. $^{10}$

Clinically, VTE can manifest as DVT and PE. In a Dutch registry, $63.6 \%$ of the VTE patients presented with DVT, whereas $32.4 \%$ of the patients suffered from PE. ${ }^{10}$ Clinically, DVT patients can present with pain, swelling, and warmth in the affected limb. Of course, DVT can embolize distal organs, particularly the pulmonary vasculature. PE can be subdivided into massive, submassive, and low-risk based on the likelihood of mortality. Low-risk PE patients may remain asymptomatic. However, patients often present with dyspnea, pleuritic chest pain, cough, and hemoptysis. The feared sequelae is hemodynamically significant PE that can lead to cardiopulmonary compromise and death. ${ }^{11}$

Apart from the immediate morbidity, VTE can also lead to long-term complications such as recurrent VTE and PTS. In the Dutch registry, $12.6 \%$ of the patients had recurrent VTE episodes. ${ }^{10}$ In comparison to the noncancer population, the rate of recurrent VTE is two- to threefold higher in the cancer population. ${ }^{12}$ Moreover, cancer patients with VTE have a two- to threefold increase in major bleeding events in comparison to the noncancer VTE patients. ${ }^{13}$ Perhaps, part of the reason is that malignant cells directly contribute to the pathogenesis of recurrent VTE. On the other hand, VTE treatment (anticoagulation) and frequent thrombocytopenia in cancer patients likely result in an increased rate of major bleeding. The increased recurrent VTE and major bleeding events have led to an increase in cancer patient mortality. ${ }^{14}$ Indeed, VTE is a leading cause of death in cancer patients receiving chemotherapy. ${ }^{15}$ Furthermore, cancer patients with VTE have markedly higher shortterm and long-term mortality rates than those without. ${ }^{16}$

Recurrent VTE is a major risk factor for PTS. ${ }^{17}$ PTS affects 20 to $50 \%$ of the DVT patients within 1 to 2 years of the index DVT episode. ${ }^{18}$ Up to $10 \%$ of the patients will develop severe PTS. ${ }^{19}$ Clinically, most PTS patients present with leg pain, heaviness, varicose veins, and/or swelling, with a minority progressing to experience skin changes and/or venous ulcers. $^{20}$ There is no gold standard test to diagnose PTS, but diagnosis and assessment of clinical severity can be aided by several scoring systems. The Clinical-Etiology-AnatomyPathophysiology (CEAP) classification is useful in characterizing the chronic venous disease. On the other hand, the PTS severity is often measured using the Villalta score, which is regarded as the international standard for the diagnosis and stratification of PTS. ${ }^{20}$ Most importantly, PTS affects cancer patients' quality of life (QOL). In a cohort study, PTS patients had significantly worse disease-specific QOL scores than those without. In addition, patients with severe PTS had more significant decrease in disease-specific QOL measures than those with the milder form of PTS. ${ }^{21}$ Persistent leg pain and swelling can prohibit cancer patients from performing basic daily tasks such as walking and standing and can lead to significant psychological burden.

\section{Pathophysiology}

The pathophysiology of GI cancer-associated VTE is complex. A thorough review of the topic is beyond the scope of this article. However, Virchow's triad dictates that the causes are broadly related to the prothrombotic state, venous stasis, and endothelial injury. GI malignancy induces a prothrombotic state by increasing tissue factor (TF) expression. ${ }^{22,23} \mathrm{TF}$ is a glycoprotein that binds to factor VII when activated. The TFfactor VII complex activates factor $\mathrm{X}$, which propagates a coagulation cascade termed the "extrinsic pathway." In addition, chemotherapy can induce tumor lysis, which releases prothrombotic, intracellular components. For example, the chemotherapeutic agent cisplatin, which is commonly used to treat colorectal and pancreatic cancer, is associated with an increased level of von Willebrand factor. ${ }^{24}$ Furthermore, chemotherapy and oncological surgery can induce direct endothelial damage. In addition, surgical oncology patients are often immobilized, which results in venous stasis.

The pathophysiology of PTS is not completely understood. The normal leg venous return is determined by the leg muscle pump and unidirectional venous valves. The cause of PTS is likely a combination of venous valve damage as a sequel of DVT, outflow obstruction, endothelial inflammation, and other factors. There is considerable debate on whether venous reflux or proximal venous occlusion plays a larger role in PTS development. ${ }^{25,26}$ The final common pathway appears to be persistent venous hypertension leading to edema, pain, and ulceration. ${ }^{27}$

\section{Management}

\section{Anticoagulation}

Anticoagulation remains the first-line therapy for GI cancerassociated VTE. Per the current standard of care, anticoagulation with low-molecular-weight heparin (LMWH) should be administered for 3 to 6 months after the index VTE incident. The safety and effectiveness of LMWH in cancer patients have been established in several randomized controlled trials. The CLOT trial, published in 2003, studied cancer patients with VTE treated with either LMWH (dalteparin) or coumarin derivative for 6 months. The authors have found that the dalteparin group had a significantly lower recurrent VTE rate ( 8 vs. $16 \%$ ) than the coumarin derivative group with similar bleeding rates (6 vs. $4 \%$ ). ${ }^{28}$ In the CATCH trial, patients with active cancer were treated with either LMWH (tinzaparin) or warfarin for 6 months. The authors found similar recurrent VTE rates between the two treatment groups ( 7.2 vs. $10.5 \% ; p=0.07$ ), whereas the warfarin group had a significantly higher rate of nonmajor bleeding (11 vs. $15 \% ; p=0.004) .{ }^{29}$ More recently published trials, such as the DALTECAN and TiCAN trials, have shown that extended LMWH treatment (6-12 months) was generally safe. ${ }^{30,31}$

Although LMWH has been shown to be superior to vitamin $\mathrm{K}$ antagonists, patients often find self-injection cumbersome. The newer direct oral anticoagulation (DOAC) agents negate the inconvenience of LMWH. Published in 2018, the Hokusai VTE Cancer Thrombosis trial investigated the effectiveness of edoxaban versus dalteparin in treating cancer-associated VTE for at least 6 months and up to 12 months. The results have shown that edoxaban was noninferior to dalteparin $(p=0.006)$. Edoxaban was associated with a statistically nonsignificant decrease in recurrent VTE rate (hazard ratio: 0.71; 
$p=0.09)$ and a statistically significant increase in major bleeding (hazard ratio: $1.77 ; p=0.04$ ). Furthermore, with regard to GI cancer, the edoxaban group had a higher rate of major bleeding compared with those treated with dalteparin (13.2 vs. $2.4 \%)^{32}$ Select-D is an ongoing trial randomizing patient to either rivaroxaban or dalteparin treatment for a total of 6 months. The first phase results have shown that the cumulative recurrent VTE rate was $11 \%$ for the dalteparin group versus $4 \%$ for the rivaroxaban group at 6 months. In addition, the major bleeding rate was $4 \%$ for dalteparin and $6 \%$ for rivaroxaban. ${ }^{33}$

Despite its safety profile and efficacy, anticoagulation is associated with an inherent risk of bleeding and recurrent VTE. In the secondary analysis of the CATCH trial, there was a $15.3 \%$ incidence rate of clinically relevant bleeding in patients treated with either LMWH or warfarin over a 6month period. ${ }^{34}$ In comparison to LMWH, DOACs have shown a similar rate of bleeding in a large, retrospective analysis ( 13 vs. $11 \%$; $p=0.746$ ). ${ }^{35}$ The anticoagulation management of GI-cancer associated VTE is even more challenging when patients are thrombocytopenic, which is relatively common when patients are treated with chemotherapy. ${ }^{36}$ Patients with thrombocytopenia are more prone to bleeding, and the anticoagulation regimen needs to be dose-adjusted and closely monitored. ${ }^{37}$ The International Society on Thrombosis and Haemostasis recommended holding anticoagulation if the platelet count is less than $25 \times 10^{9} \mathrm{~L}^{-1}$. ${ }^{37}$ Furthermore, with any medical therapy, medication compliance remains an issue, especially for patients on LMWH. Evidence has shown that more patients had to be switched from LMWH to warfarin, possibly due to the concern for selfinjection. ${ }^{38}$ The newer generation of DOAC negates many of the drawbacks of warfarin and LMWH. However, its efficacy is still limited by patient compliance.

Apart from bleeding, recurrent VTE on anticoagulation is not uncommon. In the CLOT trial, $9 \%$ of the patients treated with LMWH and $17 \%$ of the patients treated with warfarin suffered from recurrent VTE. ${ }^{28}$ In the $\mathrm{CATCH}$ trial, $7.2 \%$ of the patients in the LMWH treatment group and $10.5 \%$ of the warfarin-treated patients had recurrent VTE. ${ }^{29}$ Therefore, in cases where patients have contraindication to anticoagulation or have failed anticoagulation, an alternative form of thromboprophylaxis is required.

\section{Inferior Vena Cava Filter for GI Cancer-Associated Thrombosis}

Strong indications for inferior vena cava (IVC) filter placement include patients with symptomatic PE or proximal DVT and active bleeding or major contraindication to anticoagulation (e.g., recent surgery, intracranial metastasis, or severe thrombocytopenia). IVC filters may also be used when there is a major documented failure of anticoagulation therapy, although in some patients altering the anticoagulation regimen may be sufficient. ${ }^{39}$ Currently, the American Society of Clinical Oncology recommends IVC filter placement in conjunction with anticoagulation if there is DVT recurrence and progression despite optimal anticoagulation therapy. However, the recommendation is based on expert opinion. ${ }^{40}$
With the widespread introduction of retrievable IVC filters, filter placement is sometimes perceived as a lowrisk procedure that can protect patients from recurrent $\mathrm{PE}$. Since their advent, the number of IVC filters placed in cancer patients has expanded significantly. One study estimated that $19.2 \%$ of all cancer patients received an IVC filter during the course of the treatment. However, only $7.7 \%$ of the filters were placed in patients with an absolute contraindication to anticoagulation. ${ }^{41}$ Furthermore, it is important to realize that most retrievable filters were left in place, which can lead to multiple long-term complications such as filter fracture, migration, and perforation. The complication rates range anywhere between 2 and 20\%. ${ }^{39}$ Therefore, it is important to coordinate patient care between the interventional team and the ordering service to ensure proper filter removal once the patient can be anticoagulated. ${ }^{42}$

The primary clinical utility of the IVC filter is to prevent fatal PE. However, there exists a paucity of high-quality data examining the safety, efficacy, and mortality benefit of IVC filter in GI cancer patients. The immediate periplacement complication rate is very low. ${ }^{43}$ Further evidence has shown that IVC filter complications (filter thrombosis, migration, perforation) in cancer patients are not significantly different than that of the general public. ${ }^{44}$

With regard to filter efficacy, there are two randomized controlled trials of IVC filters in VTE patients. The PREPIC1 trial enrolled 400 patients and randomized them into either permanent filter placement with anticoagulation or anticoagulation alone. The trial has shown a decrease in recurrent PE rates at 12 days in the filter group. However, the benefit was counterbalanced with an increase in recurrent DVT rates at 2 years. ${ }^{45}$ At 8 -year follow-up, the PE protective effect persisted, whereas there was an absolute increase in recurrent DVT risk. $^{46}$ The PREPIC2 study enrolled 400 patients and randomized them into either retrievable IVC filter placement plus anticoagulation or anticoagulation alone. The authors have found that at 3 months, there was no difference in the rate of recurrent PE or mortality. However, the filter plus anticoagulation group had a significantly higher rate of recurrent DVT. ${ }^{47}$ Both PREPIC1 and PREPIC2 studies included cancer patients. However, the sample size of the cancer patients was not sufficient for stratified analysis.

Specifically, for cancer-associated VTE, Barginear et al conducted a small prospective randomized trial comparing fondaparinux with and without IVC filter placement; $25 \%$ of the patients had either colon or pancreatic cancer. The authors have found no survival benefit between the two groups at 3 years. ${ }^{48}$ Hence, for patients who can tolerate anticoagulation, the use of IVC filters is not supported by quality randomized data.

There exists a significant amount of variability in patient outcome. Brunson et al conducted a retrospective populationbased cohort study involving 14,000 patients. The presence of VTE, cancer, and IVC filter placement was identified using the ICD-9-CM (International Classification of Diseases, 9th Edition, Clinical Modification) codes. To minimize confounding variables, propensity scoring was used by applying a logistic regression model. To correct the immortal time bias, IVC filter 
insertion was used as a time-dependent covariate. The authors have found that IVC filter placement was not associated with an improvement in 30-day mortality or adjusted 180-day recurrent $\mathrm{PE}$ risk. ${ }^{49}$ On the other hand, Stein et al conducted a large retrospective study involving 266,692 patients. Patients with cancer, IVF filter placement, and PE were identified using the ICD-9-CM codes. The authors found that for patients aged $>60$ years, filter placement was associated with a significantly lower in-hospital all-cause mortality (7.4 vs. $11.2 \% ; p<0.0001)$. Furthermore, the filter group had a significantly lower 3 -month all-cause mortality (15.1 vs. $17.4 \%$; $p<0.0001){ }^{50}$ The major drawback of the study is the lack of propensity matching. Furthermore, unstable patients and those who received thrombolytic therapy were excluded from the final analysis.

Regardless, the decision to place an IVC filter in GI cancer patients must be individualized. It is reasonable to place an IVC filter when anticoagulation is absolutely contraindicated or has failed. However, the benefit of the IVC filter must be weighed against the risk of complications. ${ }^{51}$ Mansour et al have shown that patients with stage IV metastatic cancer and IVC filter insertion had a median survival of 1.31 months. ${ }^{52}$ Therefore, the benefit of IVC filter in this population may be marginal at best.

\section{Cather-Directed Thrombolysis in GI Cancer-Associated VTE}

For GI cancer-associated VTE, the standard anticoagulation therapy can prevent thrombi extension but cannot dissolve the existing clot. In contrast, the additional administration of fibrinolytic drugs actively dissolves thrombus, which may resolve venous obstruction and improve the clinical status of the limb. The safety and effectiveness of catheter-directed thrombolysis (CDT) in preventing PTS have been evaluated in a few randomized controlled trials. The CaVenT trial is widely regarded as the first rigorous randomized controlled trial. It enrolled 209 patients who were randomized to anticoagulation or anticoagulation plus CDT in treating acute proximal DVT. Primary outcomes were assessed using the Villalta score. The authors found no significant difference in the occurrence of PTS at 6 months (30.3 vs. 32.2\%; $p=0.77$ ). However, there was a significant difference at 24 months (41.1 vs. $55.6 \% ; p=0.047$ ). In the CDT group, $3.3 \%$ of the patients experienced major bleeding. ${ }^{53}$ At 5 -year follow-up, CDT was shown to be persistently superior to anticoagulation alone in preventing PTS $(p<0.0001)$, with an apparent increase in the size of the effect. However, at no time point beyond 6 months was QOL improved by use of CDT. ${ }^{54}$

A drawback of the conventional CDT lies in its prolonged exposure to lytic agents, where the treatment lasted 1 to 4 days in the CaVenT trial. Newer CDT techniques combine both the lytic agent infusion and use of mechanical thrombectomy devices. Therefore, the combination (pharmacomechanical CDT [PCDT]) decreases the patient exposure to lytic agents and is therefore theoretically safer for patients. ${ }^{55}$

The large, NIH-sponsored ATTRACT (Acute Venous Thrombosis: Thrombus Removal with Adjunctive Catheter-Directed Thrombolysis) trial enrolled 692 patients with proximal DVT and randomized them to either anticoagulation or anticoagulation plus PCDT. Patients were followed for 24 months. Patients with active cancer were excluded from this study. The authors have found that over 24 months, there was no significant difference in PTS prevention. Furthermore, major bleeding occurred in $1.7 \%$ of the PCDT group versus $0.3 \%$ in the control group $(p=0.049) .{ }^{56}$ On first glance, the ATTRACT trial may show that PCDT does not necessarily improve patient outcome. However, it is important to note that the PTS severity was significantly lower in the PCDT group from 6 to 24 months. Furthermore, on stratified analysis, the ATTRACT trial has shown the likely efficacy of PCDT in selected patient groups. For patients with femoropopliteal DVT, PCDT was not shown to improve PTS prevention, PTS severity, or QOL measurements. ${ }^{57}$ However, for patients with acute DVT involving the iliac or common femoral veins (iliofemoral DVT), PCDT was shown to provide greater reduction in presenting leg pain and swelling and to significantly decrease the PTS severity at 6 to 24 months. ${ }^{58}$ A subsequent detailed analysis of QOL found that in acute iliofemoral DVT, the use of PCDT resulted in improved VEINES-QOL score as early as 1 month post-PCDT. The differences were substantial at 1 month ( 10 points; $p<0.0001$ ) and 6 months ( 8.8 points; $p<0.0001)$. The differences were still significant but smaller at 18 and 24 months (5.8 and 6.6, $p=0.0086$ and 0.0067 in per-protocol analyses, respectively). ${ }^{59}$ On the other hand, PCDT was not cost-effective; at best, it may provide intermediate-value care for the subgroup of patients with iliofemoral DVT (\$137,000 dollars per quality-adjusted life-year). ${ }^{60}$

Finally, the Dutch CAVA Trial randomized 184 acute iliofemoral DVT patients to receive anticoagulation with or without additional ultrasound-assisted CDT. Evidence has shown that ultrasound can cause disaggregation of fibrin fibers and that ultrasound pressure waves increase lytic agent penetration into the thrombus. ${ }^{61}$ Active cancer patients were excluded from the study. The primary outcome was assessed using the Villalta score. The authors found no significant difference in terms of PTS prevention at 12 months post-CDT (odds ratio: 0.75; 95\% confidence interval: 0.38-1.5). Furthermore, no significant difference was observed in terms of venous clinical severity score or QOL. On the other hand, major bleeding occurred in four patients in the ultrasound-assisted CDT group, whereas none occurred in the standard therapy group. ${ }^{62}$

Taken together, these studies suggest that CDT and related techniques do not provide a significant clinical benefit that could justify use as the routine, first-line therapy for patients with DVT. However, in symptomatic patients with extensive thrombus (i.e., iliofemoral DVT), these procedures appear to provide better relief of presenting symptoms and may improve long-term QOL.

Despite its long history of use for DVT, CDT has not been routinely performed in cancer patients. The reason is multifold. For one, early evidence suggested that cancer patients may not derive durable benefits from CDT. Bjarnason et al have shown that the 2 -year primary patency rate was $41 \%$ in patients with malignancy compared with $75 \%$ in patients without malignancy after CDT treatment of iliofemoral DVT. ${ }^{63}$ Second, CDT has been 
associated with an absolute increase in bleeding risk. A 2016 systemic review has shown that $9 \%$ of the CDT patients experienced a major bleeding compared with $4 \%$ of the anticoagulation group. However, there was a nonsignificant difference in the rate of intracranial bleeding in this systematic review. ${ }^{64}$ Given that GI cancer patients are at an increased risk of bleeding due to the frequent thrombocytopenia and the need for anticoagulation, the absolute risk increase due to CDT needs to be taken into consideration. Furthermore, the Society of Interventional Radiology quality improvement guidelines of thrombolysis state that intracranial metastasis needs to be ruled out before CDT therapy, mostly due to the risk of fatal intracranial bleeding. ${ }^{65}$ Third, cancer patients with a short expected life span may not consider the risk of PTS to be a major priority in their overall care.

There are no prospective randomized controlled trial data focusing on the GI cancer population. However, a few retrospective studies have shown that CDT can be safe in cancer patients. Kim et al performed 202 CDT in patients with acute iliofemoral or brachiosubclavian DVT. They have found that the rate of major bleeding was $4.9 \%$ in the cancer patients and $3.4 \%$ in the noncancer patients ( $p=0.6924)$. It is noteworthy that $75 \%$ of the major bleeding events in the noncancer cohort occurred at the access site, whereas the majority of bleeding events in the cancer cohorts were GI bleeding. ${ }^{66}$ Furthermore, Brailovsky et al conducted a retrospective observational study in 1,290 cancer patients with proximal lower extremity DVT or vena-caval DVT who were treated with CDT. The authors used propensity scoring to minimize the effect of confounding variables. There was no significant difference in in-hospital mortality rate ( 1.9 vs. $2.6 \% ; p=0.23$ ) or GI bleeding rate ( 2.3 vs. $2.2 \% ; p=0.89$ ). However, the CDT group had a significant increase in the rate of intracranial hemorrhage ( 1.3 vs. $0.4 \% ; p=0.02){ }^{67}$

Although the risk of bleeding must be weighed carefully, CDT of iliofemoral DVT may be a useful therapeutic option for GI cancer patients who have severe clinical manifestations such as acute limb-threatening circulatory compromise or (more commonly) severe pain and swelling that limits ambulation despite initial anticoagulation. Patient selection remains critically important, and individual risk-and-benefit analysis must be performed before proceeding with CDT. Patients with intracranial metastasis must be excluded given the absolute increase in intracranial bleeding associated with both intracranial metastasis and CDT. In the near future, it is hoped that technical advances in thrombectomy devices may decrease the risk of CDT in GI cancer patients through reduced dose and duration of thrombolytic drug infusion. Lastly, as seen with CDT in stroke management, the improvement in institutional and technical expertise is likely to make CDT safer and more effective in managing DVT and in improving the health of cancer patients with DVT.

\section{Conclusion}

GI cancer patients with VTE are at a heightened risk for recurrent DVT and fatal PE. Anticoagulation remains the first-line therapy. For patients with symptomatic PE or proximal DVT who cannot tolerate and/or fail anticoagulation, IVC filter placement is an option for PE prophylaxis, though the existence of mortality benefit is still a matter of scientific debate. Novel endovascular therapy, such as CDT, can reduce early and late symptoms in selected, highly symptomatic patients with acute iliofemoral DVT based on the ATTRACT and CaVenT trials. Its safety profile and effectiveness may be improved with device innovations and technical improvement. The decision to proceed with endovascular interventions in GI cancer patients must be individualized, particularly with regard to symptom severity, risks, cancer stage, and life expectancy.

\section{Funding}

The authors' effort was supported by the National Center for Advancing Translational Sciences of the National Institutes of Health under Award Number TL1TR002344 (ST) and by the National Heart Lung and Blood Institute grant UH3-HL138325 for the C-TRACT Clinical Trial. The content is solely the responsibility of the authors and does not necessarily represent the official views of the National Institutes of Health.

\section{Conflicts of Interest}

Dr. Vedantham receives research grant support from Cook Medical and Medi USA.

\section{References}

1 Siegel RL, Miller KD, Jemal A. Cancer statistics, 2020. CA Cancer J Clin 2020;70(01):7-30

2 Gaba R, Lokken R. Hepatocellular carcinoma: current practice and new frontiers. Dig Dis Interv 2017 (e-pub ahead of print). Doi: 10.1055/s-0037-1603923

3 Arellano RS, Sahani DV. Imaging of cholangiocarcinoma. Dig Dis Interv 2017;1(01):8-13

4 Mauri G, Krokidis M. Ablation techniques in liver and pancreatic cancers. Dig Dis Interv 2019 (e-pub ahead of print). Doi: 10.1055/ s-0039-1692483

5 Gennaro N, Mauri G, Varano GM, et al. Thermal ablations for colorectal liver metastases. Dig Dis Interv 2019 (e-pub ahead of print). Doi: 10.1055/s-0039-1688724

6 Kim K, Shin J, ParkJH.Stenting for advanced esophageal carcinoma. Dig Dis Interv 2018 (e-pub ahead of print). Doi: 10.1055/s-0038-1645853

7 Zhou WZ, Yang ZQ. Minimally invasive interventional management of gastric outlet obstructions. Dig Dis Interv 2018 (e-pub ahead of print). Doi: 10.1055/s-0038-1639335

8 Singh R, Sousou T, Mohile S, Khorana AA. High rates of symptomatic and incidental thromboembolic events in gastrointestinal cancer patients. J Thromb Haemost 2010;8(08):1879-1881

9 Walker AJ, Card TR, West J, Crooks C, Grainge MJ. Incidence of venous thromboembolism in patients with cancer - a cohort study using linked United Kingdom databases. Eur J Cancer 2013;49 (06):1404-1413

10 Blom JW, Vanderschoot JPM, Oostindiër MJ, Osanto S, van der Meer FJM, Rosendaal FR. Incidence of venous thrombosis in a large cohort of 66,329 cancer patients: results of a record linkage study. J Thromb Haemost 2006;4(03):529-535

11 Sista AK, Kuo WT, Schiebler M, Madoff DC. Stratification, imaging, and management of acute massive and submassive pulmonary embolism. Radiology 2017;284(01):5-24

12 Lee AYY. Epidemiology and management of venous thromboembolism in patients with cancer. Thromb Res 2003;110(04):167-172 
13 Prandoni P, Lensing AWA, Piccioli A, et al. Recurrent venous thromboembolism and bleeding complications during anticoagulant treatment in patients with cancer and venous thrombosis. Blood 2002;100(10):3484-3488

14 Howlett J, Benzenine E, Cottenet J, Foucher P, Fagnoni P, Quantin C. Could venous thromboembolism and major bleeding be indicators of lung cancer mortality? A nationwide database study. BMC Cancer 2020;20(01):461

15 Khorana AA, Francis CW, Culakova E, Kuderer NM, Lyman GH. Thromboembolism is a leading cause of death in cancer patients receiving outpatient chemotherapy. J Thromb Haemost 2007;5 (03):632-634

16 Khorana AA. Venous thromboembolism and prognosis in cancer. Thromb Res 2010;125(06):490-493

17 Galanaud JP, Monreal M, Kahn SR. Epidemiology of the postthrombotic syndrome. Thromb Res 2018;164:100-109

18 Kahn SR, Ginsberg JS. Relationship between deep venous thrombosis and the postthrombotic syndrome. Arch Intern Med 2004; 164(01):17-26

19 Galanaud JP, Kahn SR. The post-thrombotic syndrome: a 2012 therapeutic update. Curr Treat Options Cardiovasc Med 2013;15 (02):153-163

20 Henke PK, Comerota AJ. An update on etiology, prevention, and therapy of postthrombotic syndrome. J Vasc Surg 2011;53(02): 500-509

21 Kahn SR, Hirsch A, Shrier I. Effect of postthrombotic syndrome on health-related quality of life after deep venous thrombosis. Arch Intern Med 2002;162(10):1144-1148

22 Shigemori C, Wada H, Matsumoto K, Shiku H, Nakamura S, Suzuki $\mathrm{H}$. Tissue factor expression and metastatic potential of colorectal cancer. Thromb Haemost 1998;80(06):894-898

23 Khorana AA, Ahrendt SA, Ryan CK, et al. Tissue factor expression, angiogenesis, and thrombosis in pancreatic cancer. Clin Cancer Res 2007;13(10):2870-2875

24 Licciardello JTW, Moake JL, Rudy CK, Karp DD, Hong WK. Elevated plasma von Willebrand factor levels and arterial occlusive complications associated with cisplatin-based chemotherapy. Oncology 1985;42(05):296-300

25 Haenen JH, Janssen MCH, van Langen $\mathrm{H}$, et al. The postthrombotic syndrome in relation to venous hemodynamics, as measured by means of duplex scanning and strain-gauge plethysmography. J Vasc Surg 1999;29(06):1071-1076

26 Roumen-Klappe EM, den Heijer M, Janssen MCH, van der Vleuten $\mathrm{C}$, Thien $\mathrm{T}$, Wollersheim $\mathrm{H}$. The post-thrombotic syndrome: incidence and prognostic value of non-invasive venous examinations in a six-year follow-up study. Thromb Haemost 2005;94(04): 825-830

27 Moustafa A, Alim HM, Chowdhury MA, Eltahawy EA. Postthrombotic syndrome: long-term sequela of deep venous thrombosis. Am J Med Sci 2018;356(02):152-158

28 Lee AYY, Levine MN, Baker RI, et al; Randomized Comparison of Low-Molecular-Weight Heparin versus Oral Anticoagulant Therapy for the Prevention of Recurrent Venous Thromboembolism in Patients with Cancer (CLOT) Investigators. Low-molecular-weight heparin versus a coumarin for the prevention of recurrent venous thromboembolism in patients with cancer. N Engl J Med 2003;349 (02):146-153

29 Lee AYY, Kamphuisen PW, Meyer G, et al; CATCH Investigators. Tinzaparin vs warfarin for treatment of acute venous thromboembolism in patients with active cancer: A randomized clinical trial. JAMA 2015;314(07):677-686

30 Francis CW, Kessler CM, Goldhaber SZ, et al. Treatment of venous thromboembolism in cancer patients with dalteparin for up to 12 months: the DALTECAN Study. J Thromb Haemost 2015;13(06): 1028-1035

31 Jara-Palomares L, Solier-Lopez A, Elias-Hernandez T, et al. Tinzaparin in cancer associated thrombosis beyond 6months: TiCAT study. Thromb Res 2017;157:90-96
32 Raskob GE, van Es N, Verhamme P, et al; Hokusai VTE Cancer Investigators. Edoxaban for the treatment of cancer-associated venous thromboembolism. N Engl J Med 2018;378(07):615-624

33 Young AM, Marshall A, Thirlwall J, et al. Comparison of an oral factor Xa inhibitor with low molecular weight heparin in patients with cancer with venous thromboembolism: Results of a randomized trial (SELECT-D). J Clin Oncol 2018;36(20):2017-2023

34 Kamphuisen PW, Lee AYY, Meyer G, et al; CATCH Investigators. Clinically relevant bleeding in cancer patients treated for venous thromboembolism from the CATCH study. J Thromb Haemost 2018;16(06):1069-1077

35 Ross JA, Miller MM, Rojas Hernandez CM. Comparative effectiveness and safety of direct oral anticoagulants (DOACs) versus conventional anticoagulation for the treatment of cancer-related venous thromboembolism: A retrospective analysis. Thromb Res 2017;150:86-89

36 Patel HK, Khorana AA. Anticoagulation in cancer patients: a summary of pitfalls to avoid. Curr Oncol Rep 2019;21(02):18

37 Samuelson Bannow BT, Lee A, Khorana AA, et al. Management of cancer-associated thrombosis in patients with thrombocytopenia: guidance from the SSC of the ISTH. J Thromb Haemost 2018; 16(06):1246-1249

38 Khorana AA, Yannicelli D, McCrae KR, et al. Evaluation of US prescription patterns: Are treatment guidelines for cancer-associated venous thromboembolism being followed? Thromb Res 2016;145:51-53

39 Li X, Haddadin I, McLennan G, et al. Inferior vena cava filter comprehensive overview of current indications, techniques, complications and retrieval rates. J Vasc Med 2020 (e-pub ahead of print). Doi: 10.1024/0301-1526/a000887

40 Key NS, Khorana AA, Kuderer NM, et al. Venous thromboembolism prophylaxis and treatment in patients with cancer: ASCO clinical practice guideline update. J Clin Oncol 2020;38(05): 496-520

41 Ho G, Brunson A, White R, Wun T. Vena cava filter use in cancer patients with acute venous thromboembolism in California. Thromb Res 2015;135(05):809-815

42 Litwin RJ, Huang SY, Sabir SH, et al. Impact of an inferior vena cava filter retrieval algorithm on filter retrieval rates in a cancer population. J Vasc Surg Venous Lymphat Disord 2017;5(05): 689-697

43 Mikhail S, Hannan L, Pishvaian MJ, Kessler C. Retrievable inferior vena cava filters in patients with cancer are safe but are they beneficial? Med Oncol 2015;32(06):622

44 Abtahian F, Hawkins BM, Ryan DP, et al. Inferior vena cava filter usage, complications, and retrieval rate in cancer patients. Am J Med 2014;127(11):1111-1117

45 Decousus H, Leizorovicz A, Parent F, et al. A clinical trial of vena caval filters in the prevention of pulmonary embolism in patients with proximal deep-vein thrombosis. Prévention du Risque d'Embolie Pulmonaire par Interruption Cave Study Group. N Engl J Med 1998;338(07):409-415

46 Decousus HPREPIC Study Group. Eight-year follow-up of patients with permanent vena cava filters in the prevention of pulmonary embolism: the PREPIC (Prevention du Risque d'Embolie Pulmonaire par Interruption Cave) randomized study. Circulation 2005; 112(03):416-422

47 Mismetti P, Laporte S, Pellerin O, et al; PREPIC2 Study Group. Effect of a retrievable inferior vena cava filter plus anticoagulation vs anticoagulation alone on risk of recurrent pulmonary embolism: a randomized clinical trial. JAMA 2015;313(16):1627-1635

48 Barginear MF, Gralla RJ, Bradley TP, et al. Investigating the benefit of adding a vena cava filter to anticoagulation with fondaparinux sodium in patients with cancer and venous thromboembolism in a prospective randomized clinical trial. Support Care Cancer 2012;20(11):2865-2872

49 Brunson A, Ho G, White R, Wun T. Inferior vena cava filters in patients with cancer and venous thromboembolism (VTE) does 
not improve clinical outcomes: a population-based study. Thromb Res 2017;153:57-64

50 Stein PD, Matta F, Lawrence FR, Hughes MJ. Inferior vena cava filters in patients with acute pulmonary embolism and cancer. Am J Med 2018;131(04):442.e9-442.e12

51 Levin JM, Schiff D, Loeffler JS, Fine HA, Black PML, Wen PY. Complications of therapy for venous thromboembolic disease in patients with brain tumors. Neurology 1993;43(06): $1111-1114$

52 Mansour A, Ismael Y, Abdel-Razeq H. Inferior vena cava filters in patients with advanced-stage cancer. Hematol Oncol Stem Cell Ther 2014;7(04):136-141

53 Enden T, Haig Y, Kløw NE, et al; CaVenT Study Group. Long-term outcome after additional catheter-directed thrombolysis versus standard treatment for acute iliofemoral deep vein thrombosis (the CaVenT study): a randomised controlled trial. Lancet 2012; 379(9810):31-38

54 Haig Y, Enden T, Grøtta O, et al; CaVenT Study Group. Postthrombotic syndrome after catheter-directed thrombolysis for deep vein thrombosis (CaVenT): 5 -year follow-up results of an open-label, randomised controlled trial. Lancet Haematol 2016;3 (02):e64-e71

55 Fleck D, Albadawi H, Shamoun F, Knuttinen G, Naidu S, Oklu R. Catheter-directed thrombolysis of deep vein thrombosis: literature review and practice considerations. Cardiovasc Diagn Ther 2017;7(Suppl 3):S228-S237

56 Vedantham S, Goldhaber SZ, Julian JA, et al; ATTRACT Trial Investigators. Pharmacomechanical catheter-directed thrombolysis for deep-vein thrombosis. N Engl J Med 2017;377(23): 2240-2252

57 Kearon C, Gu CS, Julian JA, et al. Pharmacomechanical catheterdirected thrombolysis in acute femoral-popliteal deep vein thrombosis: analysis from a stratified randomized trial. Thromb Haemost 2019;119(04):633-644

58 Comerota AJ, Kearon C, Gu CS, et al; ATTRACT Trial Investigators. Endovascular thrombus removal for acute iliofemoral deep vein thrombosis. Circulation 2019;139(09):1162-1173
59 Kahn SR, Julian JA, Kearon C, et al. Quality of life after pharmacomechanical catheter-directed thrombolysis for proximal deep venous thrombosis. J Vasc Surg Venous Lymphat Disord 2020;8 (01):8-23.e18

60 Magnuson EA, Chinnakondepalli K, Vilain K, et al. Cost-effectiveness of pharmacomechanical catheter-directed thrombolysis versus standard anticoagulation in patients with proximal deep vein thrombosis: Results from the ATTRACT trial. Circ Cardiovasc Qual Outcomes 2019;12(10):e005659

61 Engelberger RP, Spirk D, Willenberg T, et al. Ultrasound-assisted versus conventional catheter-directed thrombolysis for acute iliofemoral deep vein thrombosis. Circ Cardiovasc Interv 2015;8 (01):e002027

62 Notten P, Ten Cate-Hoek AJ, Arnoldussen CWKP, et al. Ultrasoundaccelerated catheter-directed thrombolysis versus anticoagulation for the prevention of post-thrombotic syndrome (CAVA): a single-blind, multicentre, randomised trial. Lancet Haematol 2020;7(01):e40-e49

63 Bjarnason H, Kruse JR, Asinger DA, et al. Iliofemoral deep venous thrombosis: safety and efficacy outcome during 5 years of catheter-directed thrombolytic therapy. J Vasc Interv Radiol 1997;8 (03):405-418

64 Watson L, Broderick C, Armon MP. Thrombolysis for acute deep vein thrombosis. Cochrane Database Syst Rev 2016;11:CD002783

65 Vedantham S, Sista AK, Klein SJ, et al; Society of Interventional Radiology and Cardiovascular and Interventional Radiological Society of Europe Standards of Practice Committees. Quality improvement guidelines for the treatment of lower-extremity deep vein thrombosis with use of endovascular thrombus removal. J Vasc Interv Radiol 2014;25(09):1317-1325

66 Kim HS, Preece SR, Black JH, Pham LD, Streiff MB. Safety of catheter-directed thrombolysis for deep venous thrombosis in cancer patients. J Vasc Surg 2008;47(02):388-394

67 Brailovsky Y, Yeung HM, Lakhter V, Zack CJ, Zhao H, Bashir R. Inhospital outcomes of catheter-directed thrombolysis versus anticoagulation in cancer patients with proximal deep venous thrombosis. J Vasc Surg Venous Lymphat Disord 2020;8(04):538-544.e3 